\title{
Study on Mechanism of Magnetic Abrasive Finishing Process using Low - Frequency Alternating Magnetic Field
}

\author{
Jinzhong $\mathrm{Wu}^{1, \mathrm{a}}$, Yanhua Zou ${ }^{1, \mathrm{~b}}$
}

${ }^{1}$ Graduate School of Engineering, Utsunomiya University, 7-1-2Yoto, Utsunomiya, Tochigi, Japan

a wjz.0926@163.com, byanhua@cc.utsunomiya-u.ac.jp

Keywords: Magnetic abrasive finishing, Alternating magnetic field, Finishing force, Mechanism. Abstract. The finishing force acting on workpiece surface generated by magnetic cluster is an important factor which affects cutting behavior of abrasives in magnetic abrasive finishing (MAF). In this study, we measured finishing force in alternating magnetic field and direct magnetic field, respectively. Experimental results indicated that alternating magnetic field may produce a fluctuating finishing force and the force is greater than that of direct magnetic field. We simulated the alternating magnetic force as a periodic symmetrical triangle wave and performed a Fourier transformation. The mechanism of increasing polishing depth and material removal on MAF process using alternating magnetic field were discussed.

\section{Introduction}

In the magnetic abrasive finishing (MAF) process, magnetic particles can become magnetic cluster under the influence of magnetic field. Material is removed by the relative motion between the workpiece surface and magnetic cluster [1, 2, 3]. Magnetic field is a key factor influencing the processing quality because it may generate the magnetic force necessary for finishing. In conventional plane MAF process using static magnetic field, the fine magnetic particles are easy to agglomerate during finishing process and magnetic brush is difficult to recover to its original shape after contact with workpiece surface, which hinder the realization of ultra-precision finishing to some extent. On the other hand, in complex micro surface MAF process, magnetic brush itself is still under static magnetic field, resulting abrasives cannot be transported adequately over the magnetic brush to polish into all machined surfaces.

To over these issues, we proposed an ultra-precision plane MAF process using alternating magnetic field [4]. Magnetic cluster will generate a fluctuation of up and down under the action of alternating magnetic field, which not only may prevent magnetic cluster deformation, but also promotes the abrasives into all finished surface, achieving circulation and update to ensure the stability of grinding tool. In a previous research, we investigated the magnetic field distribution in processing region [5] and discussed the effects of important process parameters namely cutting fluid, rotational speed of magnetic pole and current frequency on finishing characteristics [6]. In this paper, we studied the finishing mechanism of plane MAF process using low frequency alternating magnetic field. We investigated the reasons of increase of cutting depth and material removal, analyses relative motion between magnetic particles and workpiece and discussed the effect of alternating magnetic field on finishing trajectory.

\section{Processing Principle}

Fig.1 shows a schematic of the plane magnetic abrasive finishing process using alternating magnetic field. The tray contains the compound magnetic grinding fluid (oily grinding fluid, iron powder and abrasive), the lower is the magnetic pole and the upper is the workpiece. After electromagnetic coil entering alternating current, alternating magnetic field will be produced. A magnetic particle along magnetic equipotential line direction generates a force $\mathrm{Fx}$ and along magnetic force line direction generates a force Fy, it is calculated by (Eq. 1) [7]: 


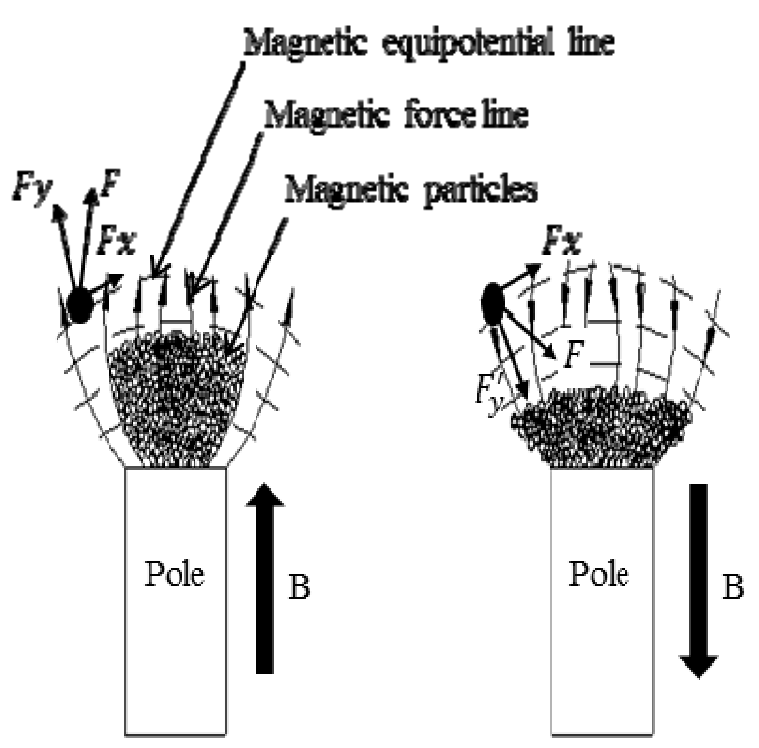

Fig.1 Force analysis of magnetic particles.

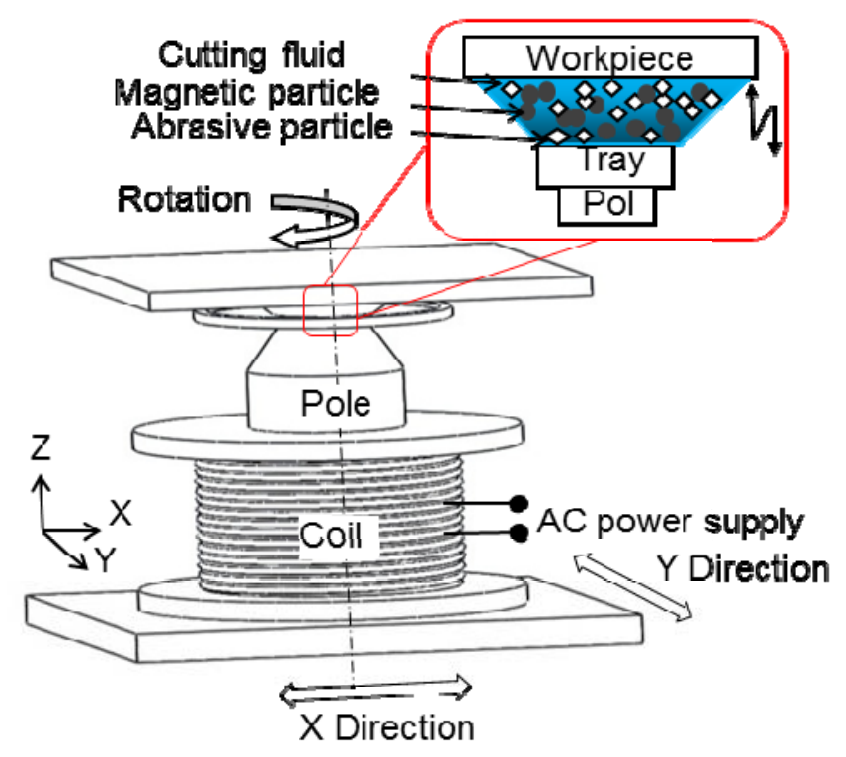

Fig.2 Schematic of processing principle.

$$
\begin{aligned}
& F_{x}=\mathrm{V} \chi \mu_{0} H\left(\frac{\partial H}{\partial x}\right) \\
& F_{y}=\mathrm{V} \chi \mu_{0} H\left(\frac{\partial H}{\partial y}\right) .
\end{aligned}
$$

Where $V$ is the volume of magnetic particle, $\chi$ is susceptibility of abrasive particles, $\mu_{0}$ is permeability of vacuum, $H$ is the magnetic field intensity, $\partial H / \partial x$ and $\partial H / \partial y$ are gradients of magnetic field intensity in $x$ and $y$ directions, respectively.

Due to the size and direction of alternating current present a cyclical variation over time, the direction of magnetic force is changing with current frequency. Fig. 2 shows the force analysis of magnetic particles in alternating magnetic field, as a result the force direction of $F y$ is changing, magnetic particles can produce the up and down movement. The movement can not only drive abrasives to float to the magnetic cluster surface for the polishing of workpiece, but also promote to the scatter of magnetic particles, achieving circulation and update to ensure the stability of grinding tools. Moreover, the connection of magnetic pole and motor can achieve the rotation and movements in all directions, thereby producing relative movement against with the workpiece to realize a wide range of finishing.

\section{Experimentation}

Experimental setup. As pictorially shown in Fig. 3, a finishing experimental device was developed for plane MAF process using alternating magnetic field. The tray is fixed on the magnetic pole so that it can achieve self-rotation by connecting the magnetic pole to a step motor. An electromagnetic coil with the wire diameter of $1 \mathrm{~mm}$ and 2000 turns has been selected to be installed on the mobile stage to achieve movement in all directions. Alternating current power device can supply the voltage and frequency in the range of $1-300 \mathrm{~V}$ and $1-999 \mathrm{~Hz}$ according to experimental needs. A finishing force measuring system had been designed. The force is measured by using two diamagnetism strain gauges (KFN-2-350-C9-11) and a data record processor (PCD-300A) (Kyowa Electronic Instrument Co. Ltd), and the measurement result is analyzed by the control software (PCD-30A).

Experimental conditions. The experimental conditions are shown in Table 1. In this study, we compared the magnetic field on finishing characteristics. The finishing time was 60 minutes. The workpiece was SUS304 stainless steel plate with the size of $80 \mathrm{~mm} \times 90 \mathrm{~mm} \times 1 \mathrm{~mm}$. In order to improve the finishing efficiency, electrolytic iron powder with the mean diameter of $30 \mu \mathrm{m}$ and aluminium 


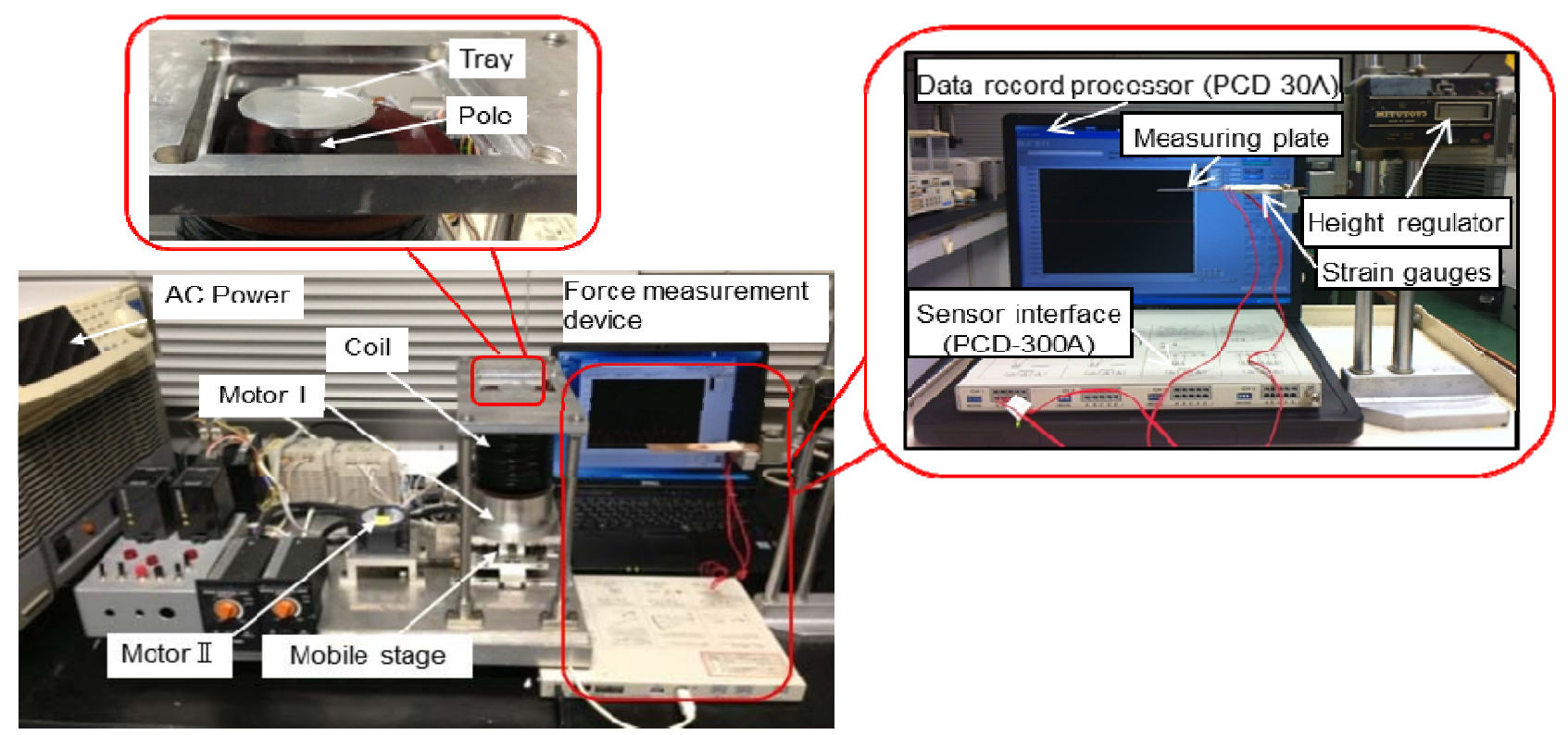

Fig.3 External view of experimental setup

oxide $\left(\mathrm{Al}_{2} \mathrm{O}_{3}\right)$ abrasive was selected in the first stage. To realize the nano-level finishing, the diameter of magnetic particle is within several microns in general. Therefore, in the second stage carbonyl iron powder with the diameter of $6 \mu \mathrm{m}$ and diamond powder with the mean diameter of $1 \mu \mathrm{m}$ were selected. The surface roughness (Ra) and surface morphology were acquired with WYKO NT1100M Veeco non-contact white light interferometer. The field of view was $0.3 \mathrm{~mm}$.

Table 1 Experimental conditions

\begin{tabular}{|l|l|}
\hline Workpiece & $\begin{array}{l}\text { SUS304 stainless steel plate with the size of } \\
80 \mathrm{~mm} \times 90 \mathrm{~mm} \times 1 \mathrm{~mm}\end{array}$ \\
\hline Finishing time & $60 \mathrm{~min}$ \\
\hline Abrasive & $\mathrm{Al}_{2} \mathrm{O}_{3}, 0-1[\mu \mathrm{m}]$ in mean dia: $0.3[\mathrm{~g}]$ \\
\hline Cutting fluid & Neat cutting oil (Honilo 988): 0.8[ml] \\
\hline $\begin{array}{l}\text { Feed speed of } \\
\text { mobile stage }\end{array}$ & $260[\mathrm{~mm} / \mathrm{min}]$ \\
\hline $\begin{array}{l}\text { Rotational speed } \\
\text { of magnetic pole }\end{array}$ & $350[\mathrm{r} / \mathrm{min}]$ \\
\hline & $\begin{array}{l}\text { Type } 1: \text { Direct magnetic field: } 1.9[\mathrm{~A}] . \\
\text { Type 2:Alternating magnetic field: } 1.9[\mathrm{~A}] \\
\text { Current frequency :3[Hz] }\end{array}$ \\
\hline
\end{tabular}

Experimental results and discussion. At first, we investigated the effect of magnetic field on finishing force, the measured results are shown in Fig. 4. In direct magnetic field, as shown in Fig. 4 (a), the forced value is a fixed value-centric with a slight fluctuations. Therefore, the direct magnetic force waveform can be considered a linear waveform with a fixed force value. As shown in Fig. 4 (b), in alternating magnetic field, a fluctuating finishing force is produced. It can be seen that the value of finishing force is greater than that of direct magnetic field from measurement results. In conventional polishing using the polisher, we can know that material removal is proportional to the finishing force, relative velocity and finishing time according to Preston equation. If the MAF also conform to Preston equation, we can conclude that this process will produce a higher material removal than that of using static magnetic cluster in the same experimental conditions. 



Fig.4. Measured finishing force wave

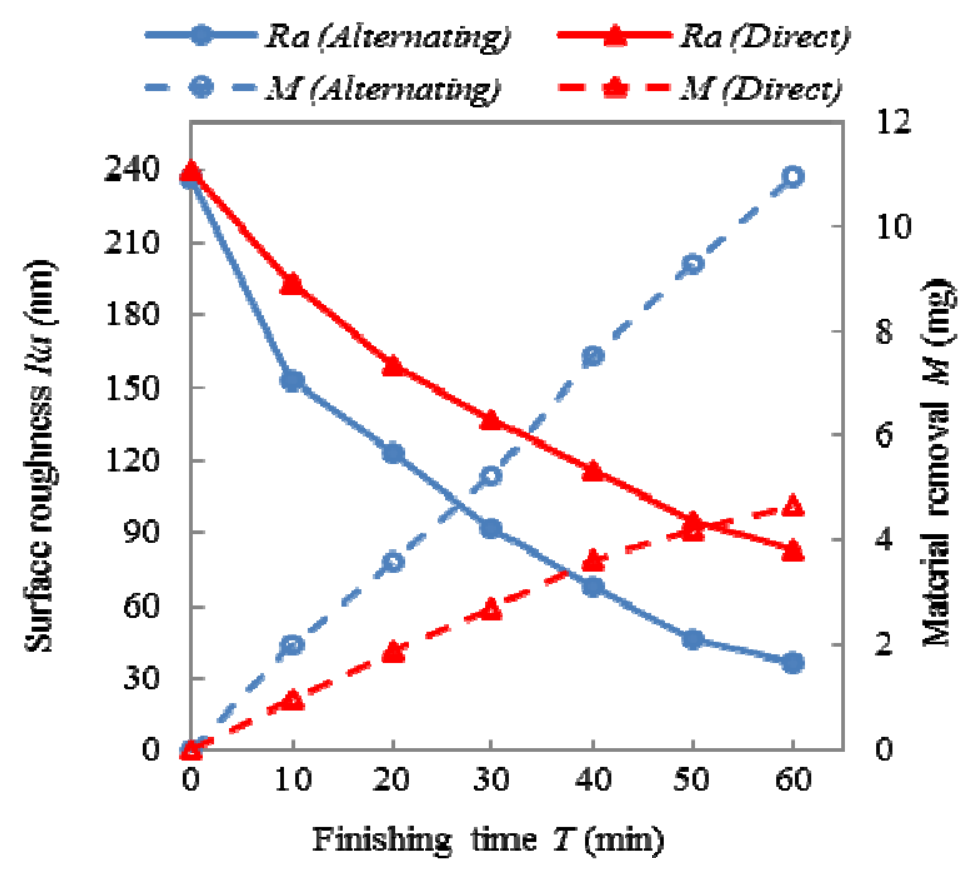

Fig.5. Effect of magnetic field surface roughness and material removal

Fig. 5 shows the variation of surface roughness $(\mathrm{Ra})$ and material removal $(\mathrm{M})$ with the finishing time in two different magnetic fields. In direct and alternating magnetic field, the material removals are $4.7 \mathrm{mg}$ and $10.95 \mathrm{mg}$, respectively. It can be seen that under alternating magnetic field material removal is approximately twice as large compared with the direct magnetic field. In alternating magnetic field, fluctuating finishing force is produced, the finishing force value increase remarkably, owing to effects of fluctuation cutting, it results in higher material removal than that in the static magnetic field.

On the other hand, in direct magnetic field, initial surface of $239.25 \mathrm{~nm}$ Ra pre-finishing was improved to $82.86 \mathrm{~nm} \mathrm{Ra}$ after finishing. In alternating magnetic field, it was improved from $236.37 \mathrm{~nm} \mathrm{Ra}$ to $36.23 \mathrm{~nm} \mathrm{Ra}$ after finishing. The surface roughness is decreasing gradually over finishing time either in alternating magnetic field or direct magnetic field. However, the improvement rate in the case of using alternating magnetic field is higher than that of the direct magnetic field. Fig. 6 shows 3D photographs of polished surfaces before and after finishing. The experimental results show that in the case of using this experimental setup MAF process using low frequency alternating magnetic field may obtain a smoother and more uniform finished surface. 


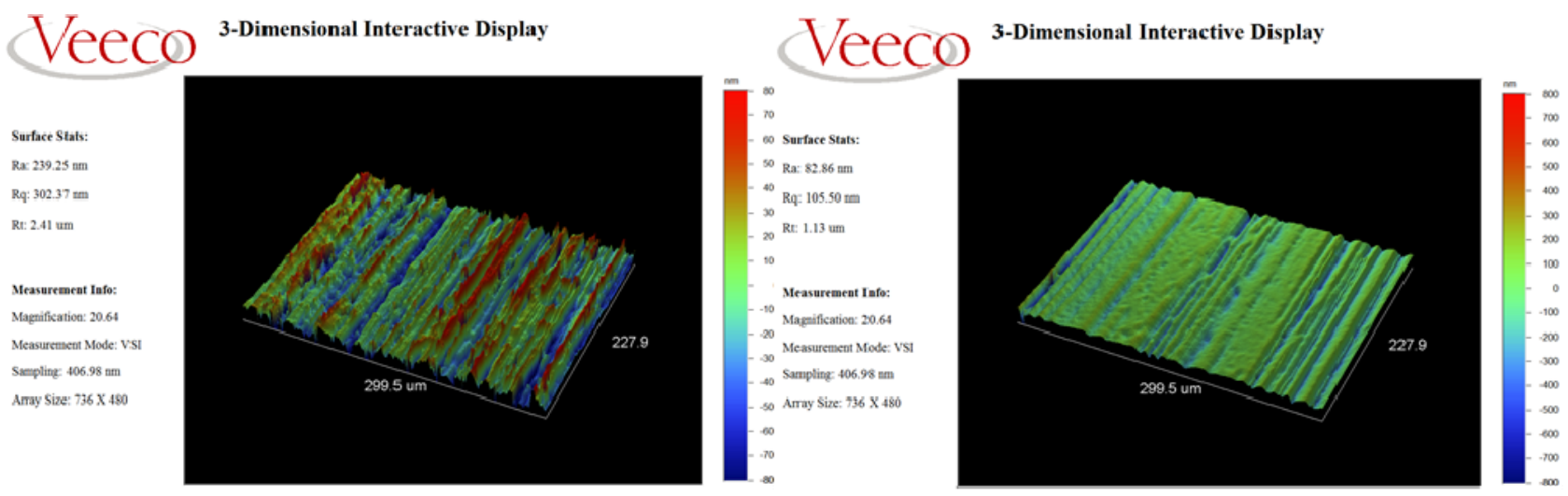

(a). Direct magnetic field

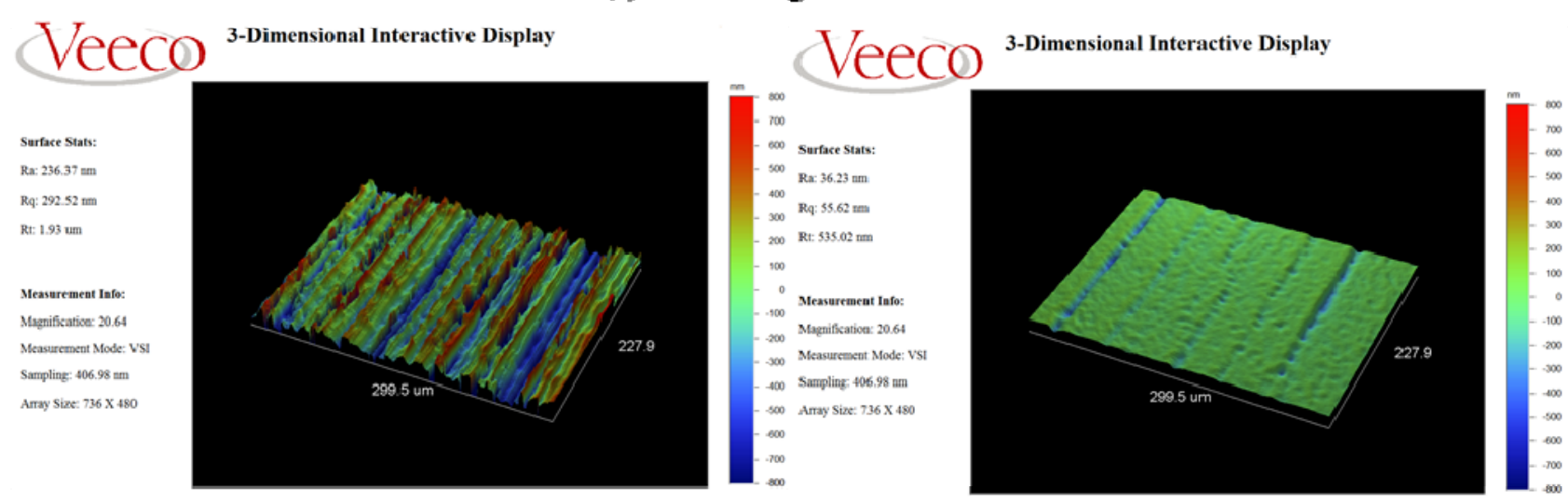

(b). Altemating magnetic field

Fig.6. 3D photographs of polished surfaces before and after finishing

\section{Analysis of finishing mechanism}

Simulation of finishing force waveform. Under direct magnetic field, the finishing force waveform is fixed value-centric and has a slight fluctuations. Under alternating magnetic field, the finishing force waveform is a continue pulse signal and presents the approximate triangle distribution. As shown in Fig. 7, in this study, we see direct magnetic force as a fixed value $P_{0}$ and simulated alternating magnetic force as a periodic symmetrical triangle wave $P(t)$. The $P(t)$ as follows [8]:

$$
=\left\{\begin{array}{ll}
\mathbf{P}(\mathrm{t}) & \left(-\frac{\boldsymbol{T}_{0}}{2} \leq \mathrm{t} \leq \mathbf{0}\right) \\
\boldsymbol{P}_{p}+\frac{\boldsymbol{P}_{p}}{\boldsymbol{T}_{0} / 2}, & \left(0 \leq t \leq \frac{\boldsymbol{T}_{0}}{2}\right)
\end{array} .\right.
$$

The fourier series expansion equation of $P(t)$ can be writen as follows:

$$
\mathrm{P}(\mathrm{t})=\frac{P_{p}}{2}+\frac{8 P_{p}}{T_{0}^{2}} \sum_{n=1}^{\infty} \frac{1-\cos \frac{1}{2} n \omega T_{0}}{n^{2} \omega^{2}} \cos n \omega t
$$

Where $P p$ is the peak value of finishing force, $T_{0}$ is the vibration period, $\omega$ is the vibration angular velocity and $t$ is time. As shown in Fig. 4, the peak value of alternating magnetic force $P p$ is $0.429 \mathrm{~N}$ and the fixed value of direct magnetic force $P_{0}$ is $0.157 \mathrm{~N}$. The peak value of alternating magnetic 
force is about 2.73 times than that of direct magnetic force. Therefore, the finishing force have a remarkably increase when we use of alternating magnetic field.

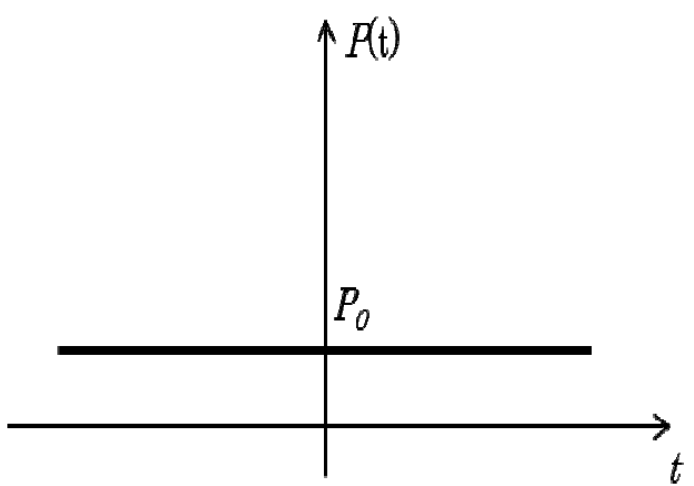

(a) Constant finishing force

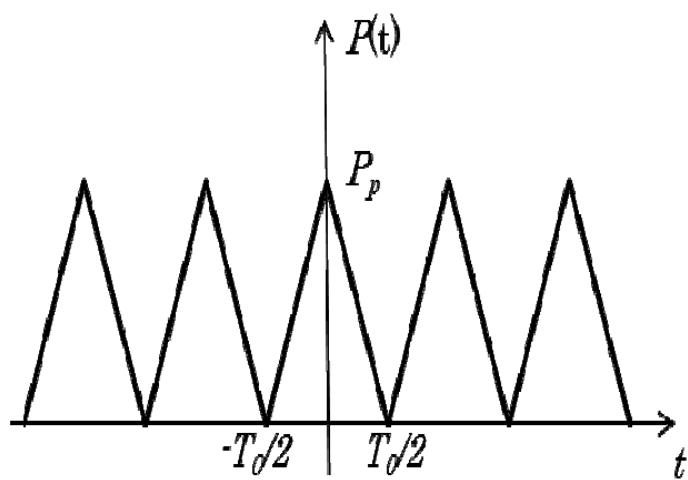

(b) Symmetrical triangular pulsed finishing force

Fig.7 Simulated diagram of finishing force

Investigation of finishing depth. The $h_{\max }$ is defined that the abrasive cutting edge produced maximal cutting depth under the maximal finishing force. We assumed that the theoretical equation of ultra-precision finishing is still appropriate for MAF process and the shape of abrasive cutting edge is cone. The relations between the maximal cutting depth $h_{\max }$ and the finishing force can be written as follows:

$$
h_{\max }=0.77 \cot \alpha\left(\frac{1}{j}\right)^{\left(\frac{1}{2}\right)}\left(\frac{P_{p}}{H_{v}}\right)^{\left(\frac{1}{2}\right)}
$$

The maximal cutting depth under alternating magnetic field $h_{\max l}$ and the direct magnetic field $h_{\max 2}$ can be obtained as follows:

$$
\begin{aligned}
& h_{\max 1}=0.77 \cot \alpha\left(\frac{1}{j}\right)^{\left(\frac{1}{2}\right)}\left(\frac{P_{p}}{H_{v}}\right)^{\left(\frac{1}{2}\right)} . \\
& h_{\max 2}=0.77 \cot \alpha\left(\frac{1}{j}\right)^{\left(\frac{1}{2}\right)}\left(\frac{p_{0}}{H_{v}}\right)^{\left(\frac{1}{2}\right)} .
\end{aligned}
$$

According to Fig. 4, we can know that the peak value of alternating magnetic force is about 2.73 times than that of direct magnetic force. Therefore, we may calculate that maximal cutting depth of alternating magnetic field is 1.65 times than that of the direct magnetic field. Due to cutting depth of abrasive cutting edge is increasing with the increase of finishing force, resulting in the rapid improvement of surface roughness.

$$
\begin{aligned}
& \frac{h_{\max 1}}{h_{\max 2}}=\left(\frac{p_{p}}{p_{0}}\right)^{\frac{1}{2}}=\left(\frac{0.429}{0.157}\right)^{\frac{1}{2}}=1.65 \\
& h_{\max 1}=1.65 h_{\max 2} .
\end{aligned}
$$

Mechanism of material removal increase. We discussed the cutting action of one abrasive that at the top of the magnetic cluster and contact with the workpiece surface in processing. In order to simplify the analysis, we assume that the shape of abrasive cutting edge is a cone and only one cutting 
edge play a role in processing when a fluctuating finishing force action on abrasive cutting edges. Therefore, the theoretical equation of ultra-precision finishing can be used.

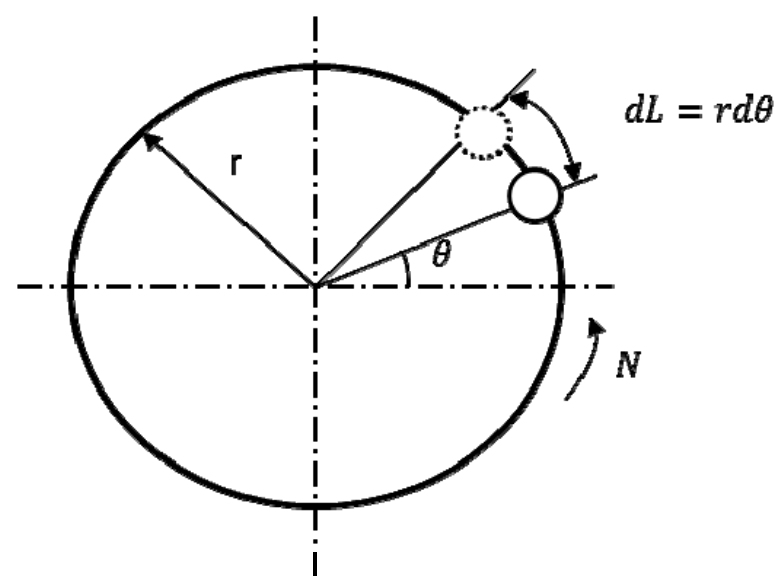

Fig.8 Rotary motion of abrasive in processing

Due to the abrasive in top of magnetic brush produce rotary motion with the magnetic pole rotation, finishing trajectory of workpiece surface presents circular. As shown in Fig. 8, $r$ is the rotation radius of abrasive, $N$ is the revolution and $\theta$ is the rotation angle. Then material removal of the micro finish length at random point can be written as follows:

$$
\mathrm{dM}=\frac{1}{C} 0.59 \rho \cot \alpha \frac{P(t)}{H V} d L .
$$

Where $C$ is coefficient, $\rho$ is the density of workpiece, $L$ is finish length, $\alpha$ is the $1 / 3$ of cone angle of the cone cutting edges and $H V$ is Vickers hardness. In static magnetic field, we set that $M_{l}$ is the material removal of an abrasive at 1/4 rotation. The expression can be written as follows:

$$
M_{1}=\frac{1}{C} 0.59 \rho \cot \alpha \frac{P_{0}}{H_{v}} \frac{\pi}{2} r .
$$

In alternating magnetic field, we set that $M_{2}$ is the material removal of an abrasive at $1 / 4$ rotation. We put the Eq. 3 into Eq. 8, the expression of $M_{2}$ can be written as follows:

$$
\begin{aligned}
M_{2} & =\int d M=\int \frac{1}{C} 0.59 \rho \cot \alpha \frac{P(t)}{H_{v}} d L \\
& =\frac{1}{C} 0.59 \rho \cot \alpha \frac{r}{H_{v}} \int_{0}^{\frac{\pi}{2}}\left(\frac{P_{p}}{2}+\frac{8 P_{p}}{T_{0}^{2}} \sum_{n=1}^{\infty} \frac{1-\cos \frac{1}{2} n \omega T_{0}}{n^{2} \omega^{2}} \cos n \omega t\right) d \theta .
\end{aligned}
$$

We introduction of a variable $\lambda$, which is the ratio between magnetic field frequency $f$ and rotational speed of magnetic pole $N$. It can be written as follows:

$$
\lambda=60 \frac{f}{N}
$$

We assume that the rotation of magnetic pole and the fluctuant of magnetic field are simultaneous and the phase difference is zero. We can obtain the relationship as follows: 
$\omega t=\lambda \theta$.

Thus, the expression of $M_{2}$ can be rewritten as follows:

$$
\begin{aligned}
M_{2} & =\frac{1}{C} 0.59 \rho \cot \alpha \frac{r}{H_{v}} \int_{0}^{\frac{\pi}{2}}\left(\frac{P_{p}}{2}+\frac{8 P_{p}}{T_{0}^{2}} \sum_{n=1}^{\infty} \frac{1-\cos \frac{1}{2} n \omega T_{0}}{n^{2} \omega^{2}} \cos n \lambda \theta\right) d \theta \\
& =\frac{1}{C} 0.59 \rho \cot \alpha \frac{r P_{p}}{H_{v}}\left(\frac{\pi}{4}+\frac{8}{T_{0}^{2}} \sum_{n=1}^{\infty} \frac{1-\cos \frac{1}{2} n \omega T_{0}}{n^{3} \lambda \omega^{2}} \sin \frac{n \lambda \pi}{2}\right) .
\end{aligned}
$$

We set $k$ is the increasing rate of material removal, which can be written as follows:

$$
\begin{aligned}
k & =\frac{M_{2}}{M_{1}}=\frac{P_{p}}{P_{0}}\left(\frac{1}{2}+\frac{16}{\pi \lambda \omega^{2} T_{0}^{2}} \sum_{n=1}^{\infty} \frac{1-\cos \frac{1}{2} n \omega T_{0}}{n^{3}} \sin \frac{n \lambda \pi}{2}\right) \\
& =\frac{P_{p}}{P_{0}}\left(\frac{1}{2}+\frac{N}{15 \pi^{3} f} \sum_{n=1}^{\infty} \frac{1-\cos \frac{1}{2} n \omega T_{0}}{n^{3}} \sin \frac{n \lambda \pi}{2}\right) .
\end{aligned}
$$

As shown in table.1, when rotational speed of magnetic pole is $350 \mathrm{r} / \mathrm{min}$ and current frequency is $3 \mathrm{~Hz}$, according to the Eq. 14, we can calculate that the increasing ratio of material removal is 2.05. Experimental results show that in direct and alternating magnetic field, the material removals are $4.7 \mathrm{mg}$ and $10.95 \mathrm{mg}$, respectively. Material removal in alternating magnetic field is approximately 2.33 times as large compared with the direct magnetic field. It can be seen that the theoretical value and practical value is nearly the same. Therefore, we may calculate that the increasing ratio of material removal under the action of fluctuating magnetic force according to the Eq. 14 .

\section{Conclusions}

1. MAF process using alternating magnetic field can produce a fluctuating finishing force which not only may prevent magnetic cluster deformation, but also promotes the abrasives into all finished surface, achieving circulation and update to ensure the stability of grinding tool.

2. In the case of using this experimental setup MAF process using low frequency alternating magnetic field may obtain a smoother and more uniform finished surface than that of direct magnetic field.

3. Based on analysis of the mechanism of increasing material removal on MAF process using alternating magnetic field, we can calculate that material removal in alternating magnetic field is approximately 2.05 times than that of direct magnetic field in this experimental setup, and the results of prediction and calculation are verified by finishing experiments.

\section{References}

[1] T. Shinmura, K. Takazawa, E. Hatano, T. Aizawa: Bull. Jpn. Soc. Precis. Eng Vol.19(1) (1985), p.54-55.

[2] Y. Zou: J. Jpn. Soc. Abras. Technol Vol. 56 (2) (2012), p. 86-89 (in Japanese).

[3] Y. Zou, T. Shinmura: J. Jpn. Soc. Abras. Technol Vol. 53(2009), p.31-34 (in Japanese).

[4] J.Z. Wu, Y. Zou: Appl. Mech. Mater Vol. 395-396 (2013), p.985-989.

[5] J.Z. Wu, Y. Zou, H. Sugiyama: J. Magnet. Magnet. Mater Vol. 386 (2015), p.50-59.

[6] J.Z. Wu, Y. Zou, H. Sugiyama: Int. J. Adv. Manuf. Technol (2015), DOI 10.1007/s 00170-015-7962-9. 
[7] M. Natsume, T. Shinmura: Trans. Jpn. Soc. Mech. Eng Vol 74 (737) (2008), p.212-218 (in Japanese).

[8] H. Matsuo: Fourier transform for engineering, Morikita Publishing limited company (2004), p.20-22. 